\title{
DETERMINATION OF THE ELECTRONIC BRANCHING RATIO OF THE $\Upsilon(9.46)$ AND AN UPPER LIMIT FOR ITS TOTAL WIDTH
}

\section{PLUTO Collaboration}

\author{
Ch. BERGER, W. LACKAS, F. RAUPACH, W. WAGNER
}

I. Physikalisches Institut der RWTH Aachen ${ }^{1}$

G. ALEXANDER ${ }^{2}$, J. BÜRGER, L. CRIEGEE, H.C. DEHNE, R. DEVENISH ${ }^{3}$,

G. FLÜGGE ${ }^{4}$, G. FRANKE, Ch. GERKE, E. HACKMACK, P. HARMS, G. HORLITZ,

G. KNIES, E. LEHMANN, B. STELLA ${ }^{5}$, R.L. THOMPSON ${ }^{6}$, U. TIMM, P. WALOSCHEK, G.G. WINTER, S. WOLFF, W. ZIMMERMANN

Deutsches Elektronen-Synchroton DESY, Hamburg

O. ACHTERBERG, V. BLOBEL ${ }^{7}$, L. BOESTEN, H. DAUMANN, A.F. GARFINKEL ${ }^{8}$,

H. KAPITZA, B. KOPPITZ, W. LÜHRSEN, R. MASCHUW ${ }^{4}$, H. SPITZER, R. VAN STAA,

G. WETJEN ${ }^{9}$

1I. Institut für Experimentalphysik der Universität Hamburg ${ }^{1}$

A. BÄCKER ${ }^{10}$, K. DERIKUM, C. GRUPEN, H.J. MEYER, B. NEUMANN, G. ZECH

Gesamthochschule Siegen ${ }^{1}$

and

H.J. DAUM, H. MEYER, O. MEYER, M. RÖSSLER, K. WACKER ${ }^{10}$

Gesamthochschule Wuppertal ${ }^{1}$

Received 7 March 1980

Revised manuscript received 23 April 1980

We have measured the reaction $\mathrm{e}^{+} \mathrm{e}^{-} \rightarrow \mathrm{e}^{+} \mathrm{e}^{-}$at the $\Upsilon$. Using the excess of electron pairs over the QED expectation we obtain an electronic branching ratio of $B_{\mathrm{ee}}=(5.1 \pm 3.0) \%$. Combining this with published values of the muonic branching ratio $B_{\mu \mu}$ we conclude that the $\Upsilon$ is a narrow state with a total width $<180 \mathrm{keV}$ at $95 \%$ C.L.

After the discovery of the $\Upsilon$ family in protonnucleus collisions [1], $\mathrm{e}^{+} \mathrm{e}^{-}$-experiments have determined precisely the mass and the leptonic width of the $\Upsilon(9.46)[2-4]$. However only lower limits for the total width could be given thus far, due to the large errors in the muonic branching ratio measurements $[2,5,6]$.

In the present paper we report on an analysis of the reaction $\mathrm{e}^{+} \mathrm{e}^{--} \rightarrow \mathrm{e}^{+} \mathrm{e}^{-}$at the $\Upsilon$. Using the evaluation techniques for Bhabha scattering developed for
${ }^{1}$ Supported by the BMFT, Germany.

2 On leave from Tel Aviv University, Israel.

${ }^{3}$ Now at Oxford University, England.

${ }^{4}$ Now at Universität and Kernforschungszentrum, Karlsruhe, Germany.

${ }^{5}$ On leave from University of Rome, Italy; partially supported by INFN.

${ }^{6}$ Permanent address: Humboldt University, Arcata, California, USA.

${ }^{7}$ Now at CERN, Geneva, Switzerland.

8 Permanent address: Purdue University, W. Lafayette, In., USA.

${ }^{9}$ Now at Scientific Control Systems GmbH, Hamburg.

10 Now at Harvard University, Cambridge, Mass. USA. 
testing QED at PETRA energies [7], we determine the $\Upsilon$ branching ratio into electrons from the excess of electron pairs over the QED expectation. Assuming $\mu$-e universality, we obtain the branching fraction of the $\Upsilon$ into lepton pairs, $B_{l l}=\Gamma_{l l} / \Gamma_{\text {tot }}$. From this we derive the total width $\Gamma_{\text {tot }}$ of the $\Upsilon$ using the electronic width published previously [2]

The experiment was performed with the detector PLUTO at the $\mathrm{e}^{+} \mathrm{e}^{-}$storage ring DORIS [7]. Data were taken on the $\Upsilon$ from 9.450 to $9.465 \mathrm{GeV}$. The electron pairs were identified by means of the inner track detector and the surrounding shower counters. Events were accepted if both electrons deposited more than a third of the beam energy in the shower counters and if they were collinear to within $15^{\circ}$ in spatial angle. We obtained a final sample of 2953 events. The total efficiency for recognizing an $\mathrm{e}^{+} \mathrm{e}^{-}$ $\rightarrow \mathrm{e}^{+} \mathrm{e}^{-}$event was determined to be $99.3 \pm 0.4 \%$.

The acceptance in scattering angle $\theta$ was restricted to the region $|\cos \theta|<0.8$, where charge identification was possible. The probability of misidentifying the charges of both electrons was determined using the small sample of electron pairs where both tracks had the same charge assignment. The corresponding event numbers for different regions of $|\cos \theta|$ are given in table 1 . This point is of particular relevance since the effect of the resonance decays shows up mainly in the backward region where misinterpreted electron pairs from the forward direction could fake a higher electronic branching ratio. For the final $\cos \theta$ distribution the electron pairs with the same charge assignment were divided up according to the measured ratio of events in the corresponding forward and backward bins. The remaining charge misidentification probability is negligible.

Table 1

Charge misidentification probability.

\begin{tabular}{lcc}
\hline $\cos \theta \mid$ & $\begin{array}{l}\text { No. of Bhabhas with } \\
\text { unique sign of } \cos \theta\end{array}$ & $\begin{array}{l}\text { No. of Bhabhas with } \\
\text { ambiguous sign of } \cos \theta \\
\text { from the momentum } \\
\text { measurement }\end{array}$ \\
\hline $0-0.3$ & 338 & 9 \\
$0.3-0.5$ & 406 & 7 \\
$0.5-0.7$ & 893 & 29 \\
$0.7-0.8$ & 1211 & 60 \\
sum & 2848 & 105 \\
\hline
\end{tabular}

The main background sources were (i) reactions of the type $\mathrm{e}^{+} \mathrm{e}^{-} \rightarrow \gamma \gamma$ where both photons converted in the innermost part of the detector, (ii) the reaction $\mathrm{e}^{+} \mathrm{e}^{-} \rightarrow \tau^{+} \tau^{-} \rightarrow \mathrm{e}^{+} \mathrm{e}^{-} \nu \bar{\nu} \nu \bar{\nu}$, (iii) cosmic showers and (iv) beam-gas interactions. Five events of the last two classes were removed from the sample of backward scatters by a visual scan. The total background resulted to $(0.5 \pm 0.2) \%$ and was subtracted. For more details of the analysis see ref. [7].

Fig. 1 shows the resulting differential Bhabha cross section in the resonance region $\left(9.45 \leqslant E_{\mathrm{cm}} \leqslant 9.465\right.$ $\mathrm{GeV}$ ). The full curve is the first order QED cross section modified by radiative corrections [8] and the angular resolution. Although QED describes the overall behaviour of the resonance data quite well, fig. 1 shows a small excess of events in the backward direction $(\cos \theta<0)$. Here a contribution from $\Upsilon \rightarrow$ ee decays is expected to show up.

In the presence of a resonance the QED Bhabha cross section has to be modified by adding a resonance

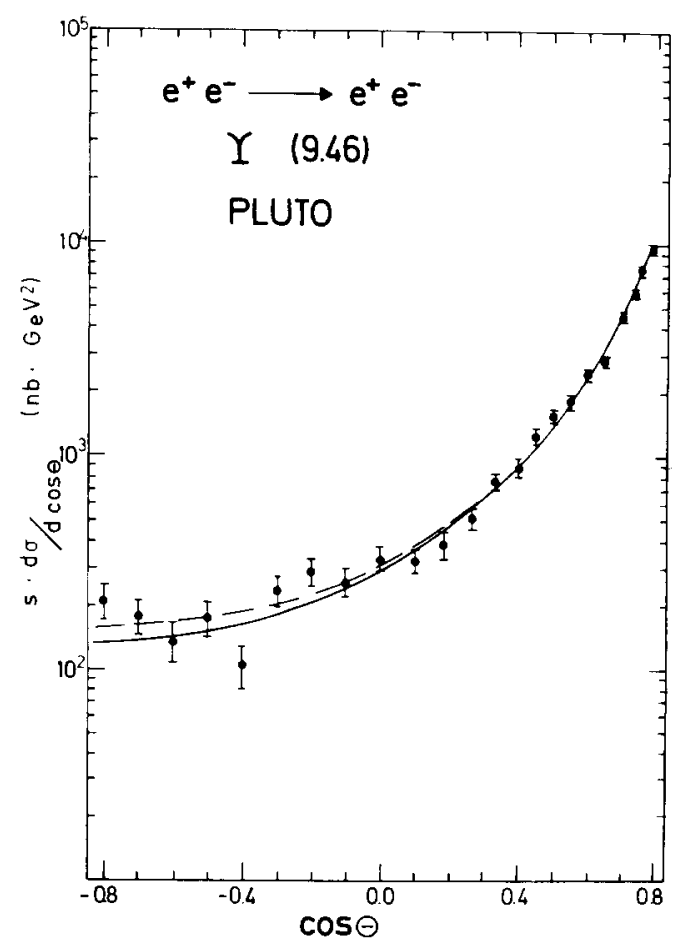

Fig. 1. The differential cross section $s \mathrm{~d} \sigma /(\mathrm{d} \cos \theta)$ for reaction $\mathrm{e}^{+} \mathrm{e}^{-} \rightarrow \mathrm{e}^{+} \mathrm{e}^{-}$on the resonance $\left(9.45 \leqslant E_{\mathrm{cm}} \leqslant 9.465\right)$. The full curve is the QED part including the effects of radiation and of finite angular resolution. The dashed curve also includes the direct decay $\Upsilon \rightarrow \mathrm{e}^{+} \mathrm{e}^{-}$. 
contribution and an interference term. Since we integrate over the central part of the resonance region from 9.45 to $9.465 \mathrm{GeV}^{\ddagger 1}$ the interference effect does not contribute to first order. The angular distribution of the $\Upsilon \rightarrow$ ee decay is expected to be of the form $1+\cos ^{2} \theta$, i.e. it is symmetric in $\cos \theta$. The dashed curve in fig. 1 shows the result of fitting the sum of the resonant and the Bhabha distribution to the data, each with one free scale parameter. The fit gives a total cross section for ee $\rightarrow \Upsilon \rightarrow$ ee of $(0.53$ $\pm 0.31) \mathrm{nb}$. Using the theoretical angular distribution as a reference is justified by the good agreement between QED and experiment at $9.4 \mathrm{GeV}$ and also at higher energies [7]. In fact, a fit which uses only the measured on-and off-resonance cross sections leads to $(0.42 \pm 0.43) \mathrm{nb}$, consistent with the more precise value above.

The cross section has so far not been corrected for radiative effects. We therefore compare it to the corresponding uncorrected cross section for hadrons [9] $\sigma_{\text {tot }}$ in order to eliminate the effects of radiation in the initial state. We estimate that final state radiation affects the result by less than $5 \%$, and other systematic uncertainties by less than $15 \%$. The resulting electronic branching ratio, including all errors, is $B_{\mathrm{ee}}$ $=(5.1 \pm 3.0) \%$.

This value is compatible with the muonic branching ratio $B_{\mu \mu}=(2.3 \pm 1.4) \%$ (average value from refs. [2] and [5]), and therefore with $\mu$-e universality in these decays. Assuming lepton universality to hold, we can take the weighted mean of all, $B_{l l}=(2.9 \pm 1.3) \%$, as the best estimate of the leptonic branching ratio.

The larger expectation value and the smaller error of $B_{l l}$ now lead to a nontrivial upper limit on the total width $\Gamma_{\text {tot }}(\Upsilon)$. Using our published electronic width $\Gamma_{\text {ee }}=(1.33 \pm 0.14) \mathrm{keV}[2]$, we obtain $1 / \Gamma_{\text {tot }}$ $=(22 \pm 10) \mathrm{MeV}^{-1}$, and in particular $\Gamma_{\text {tot }}<180 \mathrm{keV}$ at $95 \%$ C.L. This limit is two orders of magnitude lower than the previous ones obtained from the beam energy spread of DORIS. The best estimate ${ }^{\ddagger 2}$ for the

$\neq 1$ This corresponds to an interval of $2 \sigma\left(E_{\mathrm{cm}}\right)$, where $\sigma\left(E_{\mathrm{cm}}\right)$ is the r.m.s. energy spread of the storage ring.

$\neq 2$ Note that the branching ratios as well as $1 / \Gamma_{\text {tot }}$ have normally distributed errors, but $\Gamma_{\text {tot }}$ does not. total width, $45_{-14}^{+38} \mathrm{keV}$, is well compatible with QCD expectations $[10]$.

In conclusion, our result shows that the $\Upsilon$ is indeed a narrow state with a total wid th comparable to that of the $J / \psi$. This observation confirms the current idea of the $\Upsilon$ being a heavy quarkonium state.

We are grateful to D. Degele and his colleagues at DORIS, whose outstanding efforts made these measurements possible. We are indebted to all the service groups which supported the experiment, namely the computer center, the synchroton staff, the gas supply group and the vacuum group. Our special thanks go to our technicians, who have constructed most of the detector parts and taken care of it during running times. The non-DESY members of the PLUTO group want to thank the DESY directorate for support and hospitality extended to them. Part of this work was supported by the Bundesministerium für Forschung und Technologie.

\section{References}

[1] S.W. Herb et al., Phys. Rev. Letters 39 (1977) 252; W. Innes et al., Phys. Rev. Letters 39 (1977) 1240.

[2] PLUTO Collaboration, Ch. Berger et al., Zeitschrift für Physik C1 (1979) 343.

[3] C.W. Darden et al., Phys. Letters 76B (1978) 246.

[4] J.K. Bienlein et al., Phys. Lett. 78B (1978) 360.

[5] C.W. Darden et al., Phys. Lett. 80B (1979) 419.

[6] G. Heinzelmann, Proc. of the XIX Intern. Conf. on High Energy Physics, Tokyo (1978) Physical Society of Japan 1979 , p. 263.

[7] PLUTO Collaboration, Ch. Berger et al., Test of QED in the reactions $\mathrm{e}^{+} \mathrm{e}^{-} \rightarrow \mathrm{e}^{+} \mathrm{e}^{-}$and $\mathrm{e}^{+} \mathrm{e}^{-} \rightarrow \mu^{+} \mu^{-}$at cms energies from 9.4 to $31.6 \mathrm{GeV}$, DESY report $80 / 01$ (1980), to be published in Zeitschrift für Physik C; B. Koppitz, Thesis, Internal Report DESY PLUTO 80/ 05 (1980).

[8] F.A. Berends et al, Nucl. Phys. B63 (1973) 381, Nucl. Phys. B68 (1974) 541, Phys. Lett. 63B (1976) 432.

[9] C. Gerke, Thesis, Internal Report DESY PLUTO 80/03 (1980), unpublished.

[10] T. Appelquist and H.D. Politzer, Phys. Rev. Lett. 34 (1975) 43.

We thank Dr. Z. Kunszt for information about higher order QCD corrections. 